\title{
Research on Optimal Routing Real-time Scheduling Algorithm for CPS
}

\author{
Zhou Benhai1 ${ }^{1, a}$, Liu Limei ${ }^{1}$ Xu Libo ${ }^{1}$ \\ ${ }^{4}$ Department of Computer Science, Shenyang Institute of Engineering, Shenyang City, Liaoning \\ Province, 110136, China \\ a13734610r@qq.com.
}

keywords: routing;real-time scheduling;wireless sensor networks

Abstract. CPS is an integrated system of physical process and calculation process. Through the CPS system, human beings are related by the digital world and the physical world. The CPS sensing layer is mainly composed of wireless sensor networks. At the beginning of the data collection task, the sensor nodes are randomly or manually assigned to the monitored location. When the nodes collect the required environment information, the information is transmitted to the terminal node through the self-group routing path. The terminal node transmits all the information collected by the satellite or other methods to the receiving terminal that can be observed by the detector. In the application of CPS wireless sensor networks, the self-routing strategy can greatly affect the performance of the network. Therefore, it has been one of the key issues of the research. According to the delay and working time of the wireless sensor networks, we propose a new real time routing protocol. The algorithm can reduce the network congestion and balance the load of each node effectively.

\section{Introduction}

CPS is an integrated system of physical processes and computational processes. The role of objects includes all aspects of the real world: natural environment, construction, machinery, but also includes human beings and so on. Through the CPS system, human beings are related by the digital world and the physical world. Wireless sensor network is the most important infrastructure for CPS.

The function of wireless sensor network is to collect the data acquisition, processing and communication for the application of network. With the rapid development of hardware manufacturing technology and wireless communication technology, the sensor nodes have the characteristics of small size, low cost, high precision of data acquisition and so on. Generally speaking, the deployment cost of wireless sensor network is low, and the information collection speed is fast. As a result, it is widely used in the field of early warning, detection, and building condition monitoring.

Wireless sensor network has brought great convenience for modern industry, agriculture and people's daily life. Wireless sensor network technology has broad application prospects, so it is recognized as one of the most important applications.

The most important characteristic of wireless sensor networks is the large number of sensor nodes. A sensor node is usually composed of a sensor module, a processor module, a wireless communication module and an energy supply module. Sensor nodes usually have the characteristics of miniaturization, intelligence, autonomy and diver. With the development of manufacturing technology, the size of the sensor has changed very small, good anti-jamming, and characteristics of resistance to physical and chemical damage. So, the WSN nodes cannot occupy a space limited to locations. Otherwise, wireless sensor net-works are characterized by intensive, flexible, selforganizing and multi hop.

\section{Cps Routing Policy}

Generally, wireless sensor network routing policy can be divided into three types: flat-class routing, hierarchical routing and routing based on location. The following three types of routes will introduce specific policies. 
2.1 Level Routing. Same level routing can be called same level multi-hop routing in the same level routing. In the same level routing, each node plays the same role, including the collection of information, forwarding information, and forwarding information in the pro-cessing of the base station. In the same level routing, there is no relationship between the upper and lower levels of each node, and there is no coordination node as a temporary data aggregation node. A typical same level routing concludes the SPIN, Directed Diffusion, MCFA and GBA.

2.2 Hierarchical Routing. Hierarchical routing is sometimes called cluster-based routing structure. In hierarchical routing, there are usually individual nodes with a strong computing power and more power supply. These nodes often take on more work, such as collecting information gathering its neighbour nodes and then forwarded to the base station, etc. while the rest of the different nodes in general are only responsible for the collection of information. Hierarchical routing process is generally relatively simple. Common hierarchical routing protocols concludes LEACH, TEEN, APTEEN, VGA and other agreements.

2.3 Location-Based Routing. The location-based routing policy is based on the relative position or absolute position of the node. Using the routing protocol in the application scenario, the sensor nodes are usually based on the wireless signal strength, relative coordinates or GPS information absolute coordinate information, which can obtain a more accurate routing path.

Due to the application of the known location in the scenario, the routing path is relatively easy to obtain, and relatively stable and fixed. Therefore, the routing protocol based on the geographical position can save energy by the node's regular sleep strategy, which can prolong the network lifetime. The design goal is to make the node as far as possible in the state of sleep without data transmission. Generally, location-based routing protocols conclude GAF, LCR and GPSR etc.

Through the above summary, we can see the wireless sensor network routing algorithm design and its application environment and the scene has a close relationship. Each routing algorithms are based on specific application scenarios for the design goal, because there is no algorithm can take into account a number of characteristics of wireless sensor net-works. So the clear requirement of the application is the basic condition of wireless sensor network routing design.

\section{An Optimal Routing Real-Time Scheduling Algorithm for Cps}

3.1. The Model of CPS Routing Policy. The main application of the sensor network is to collect information about the changes of the surrounding environment. In this paper, static sensor network collects information for the application of the scene as the research object. In this scenario, the two features of the sensor network are important indicators to measure the merits of the routing. The first indicator is the delay of information collection. When the node is aware of the information, the packet is delivered to the sink node. If there is a serious traffic congestion in the network, the transmission delay will be very high. Therefore, we can use the network congestion state to measure the delay of network da-ta transmission. The second index is the working time of the sensor network. Through these two indexes, we propose a hierarchical routing algorithm with multiple paths. This algorithm can make full use of the forward link between the nodes, so as to reduce the network congestion caused by the data forwarding to the sink node. At the same time, the algorithm can effectively balance the load of each node, prevent the individual nodes from working in the condition of energy depletion, and further extend the life of the network.

Let $\mathrm{v} 1 \ldots \mathrm{v} 2$ be a node in the sensor network. v0 is the sink node (the gateway node, which is referred to as the sink node). All nodes are randomly deployed in the observation area of $M * M$. We can use an undirected connected graph $\mathrm{G}(\mathrm{V}, \mathrm{E})$ to represent the topology of nodes, where $\mathrm{V}$ is the set of all points, and $E$ is the set of all sides. If the two sensor nodes vi and vj within the communication range of each other, while (vi, vj) is the number of edges. Because our study mainly focuses on static sensor networks, so we assume that the wireless sensor network has the following characteristics:

1)The network is static, which means that the sensor nodes and sink nodes after deployment is fixed; 
2)All nodes have the same initial energy, the sink node has unlimited energy supply and adequate computing power;

3) Each node has the same and limited transmission distance, so most of the nodes can-not communicate directly with the sink node.

3.2 The Algorithm Description. In the topology of the new routing method, data packets are forwarded to the sink nodes from the higher nodes to the lower nodes. Assuming that the level of a node is L, it can only be sent to the L-1 node or L node. Each node has multiple paths to reach the sink node.

According to our previous analysis, path routing selection problem is actually the balance node and reducing the node forwarding queue waiting time. The method chooses the next hop forwarding node, that does not exist some nodes in the pending queue longer which increases the packet transmission delay or some nodes for forwarding times too much, so as to premature depletion of energy to stop working.

If we randomly sends $\mathrm{n}$ data packets to the neighbor node of the routing table, we assume there are $\mathrm{m}$ nodes (for the convenience of expression, we assume that $\mathrm{m}=\mathrm{n}$ in the routing table. The following conclusions when $\mathrm{m}=\mathrm{n}$ can also set up to launch). Then, the probability $\mathrm{P}[\mathrm{i}, \mathrm{k}]$ of routing table specific node i received data packets is as follow:

$$
p[i, k]=\left(\begin{array}{l}
k \\
n
\end{array}\right)(1 / n)^{k}(1-1 / n)^{n-k}
$$

The $\mathrm{k}$ information packets are sent to the same neighbor node $\mathrm{i}$. The probability is $(1 / \mathrm{n}) * \mathrm{k}$. if we consider the routing table in the specific node $\mathrm{i}$ at least received $\mathrm{K}$ packets, the probability $\operatorname{pr}[\mathrm{i}, \mathrm{k}]$ formula is as follows:

$$
\operatorname{pr}[i, k]<\left(\begin{array}{l}
k \\
n
\end{array}\right)(1 / n)^{k}
$$

We found that the choice of two nodes is better than that only selecting a node of the. Similarly, we can let two choices two become $m$ choices. And then, the nodes compare their received the number of packets in order to select smallest number of the node to forward packets. As a result, the expect packet number is as follow:

$$
E_{\max \text { packets }}=\ln \ln n / \ln m
$$

From the above formula can be drawn, the results of the route are not optimized a lot. As a result, the new CPS routing scheduling algorithm is described as follows:

1) We define the number of nodes that have been forwarded to be $\mathrm{N}$.

2) The initial $\mathrm{N}$ of the node is set to 0 . When a node receives a packet, it updates its $\mathrm{N}$ value to all its child nodes.

3) When a node wants to forward data packets, it will randomly select two parents or peer nodes in their own routing table, and compare their $\mathrm{N}$ values.

4) The node selects the next hop node as the $\mathrm{N}$ value of the smaller node. This node does not have to compare all the nodes in the routing table at each time. Moreover, when the number of the next hop node is selected, the forwarding processing time can be reduced. At the same time, this selection strategy based on the previous calculation can meet the average node number of transmission purposes.

\section{Experiment Results Analysis}

We use $\mathrm{C}++$ to prepare the node forwarding and receiving system. In the experiment, we have 80 nodes randomly deployed in a $10 \mathrm{~km} * 10 \mathrm{~km}$ square area. Sink nodes are deployed in the lower left corner of the area, and the absolute coordinates are $(0,0)$. The maximum transmission distance of the sensor node is defined as $2 \mathrm{~km}$. Figure 3.3 compares the tree topology and MPHR topology in the same node position. The black point represents the sensor node. The blue point represents the sink node. The red line indicates the path of the selected packet for-warding. 
In the experiment, each node is assigned to the initial energy of $100 \mathrm{~J}$. We assume that each computation path and forwarding packets need to consume the energy of $0.1 \mathrm{~J}$. We let each node generates a 20 bytes packet, and the data packet through the new routing strategy is forwarded to a sink node. In the experiment, we are setting up a wireless network size of 100 nodes, 120 nodes, 140 nodes, 160 nodes, 180 nodes and 200 nodes. When the number of nodes in different deployment area under the same degree of different node.

In the experiment, we compared two routing strategies: the single path routing strategy based on tree topology and the new routing strategy. The new routing algorithm in the selection of random selec-tion of two nodes compares their number of for-warded packets $\mathrm{N}$. In the end, the smaller $\mathrm{N}$ nodes are used as the next hop nodes.

4.1. The Comparison of Energy. In the experiment, we define the lifetime of the sen-sor network as the first node in the network to run out of energy as a result of fail working. In Figure 1, we draw a graph of the number of nodes in the sen-sor network lifetime (unit 10000s). It can be seen that the new routing strategy compared to the tree routing strategy can significantly prolong the net-work lifetime. At least, the new routing method can increase the network lifetime up to $55.3 \%$.

Figure 1. The comparison of energy with two routing methods.

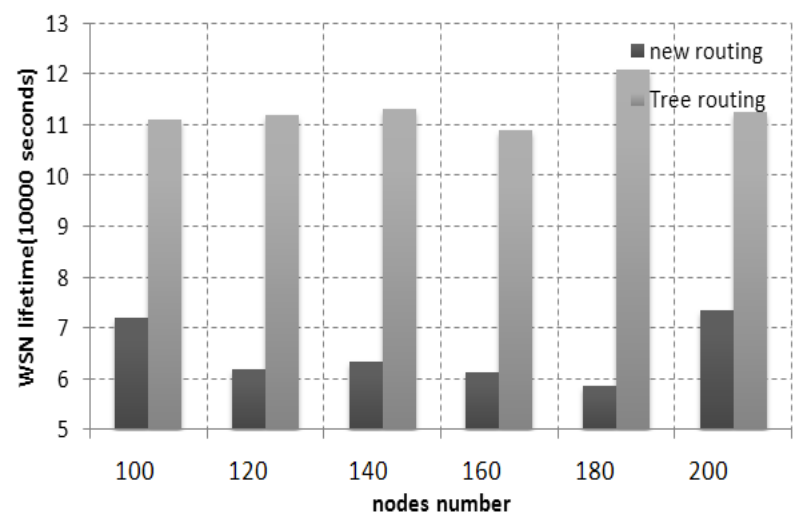

4.2. The Comparison of Energy Comparison of Congestion. In this experiment, we assume that when a sensor node in the processing of the data packet, it cannot accept packets from other nodes to forward the data. If this packet to be transmitted, a packet is as dis-card processing. Therefore, the packet loss rate (we refer to the proportion of the total number of times) to a certain extent, denotes the degree of congestion of the network. Table two compares the packet loss rate of the two routing strategies. It can be seen that the new routing strategy has a very low packet loss rate compared to the tree topology routing strategy. This is because the multipath transport topology provides redundant transmission paths, so that packets can be distributed to different nodes for transmission.

Figure 2. The comparison of packet loss rate with two routing methods.

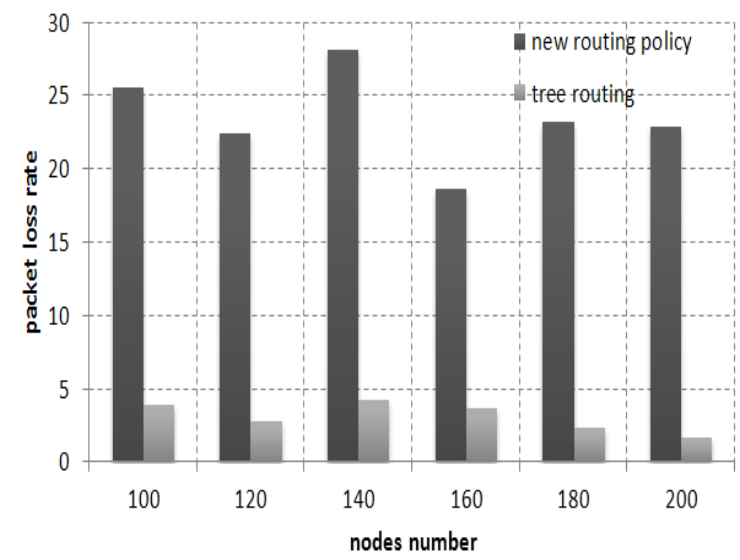


According to the simulation results, we can draw the conclusion that MPHR is a routing strategy for static wireless sensor networks which is suitable for data collection. Compared with the commonly used routing strategy based on tree topology, it can significantly prolong the network lifetime and greatly reduce the congestion of the network.

\section{Conclusion}

The CPS sensing layer is mainly composed of wire-less sensor networks. In the data collection task, sensor nodes are random or artificial planning layout in the monitored locations. The node collected the required environmental information, which will be transmitted to the terminal node through the routing path from the group. In this chapter, we propose a multi path routing strategy. In this paper, we propose a multi path routing strategy. This strategy can make full use of the forwarding relationship between nodes. By using the redundant forwarding path, the routing strategy reduces the average forwarding times of nodes and prolongs the lifetime of the net-work.

\section{Acknowledgement}

This paper is supported by Research foundation of Liaoning Provincial Education Department (L2014532, L2014520, L2014523), National Social Science Fund(15CGL050).

\section{References}

1. Jonathan W. Hui, David E. IP is Dead, Long Live IP for Wireless Sensor Networks. 5-7(2008).

2. Vikrant Saigal, Ajit K. Nayak, Sateesh K. Pradhan, R. Mall, Load balanced routing in mobile ad hoc networks, Computer Communica-tions.157-168 (2004).

3. Xiaoyuan Hong, Mario Gerla and Hanbiao Wang, Load Balanced.Energy-Aware Communications for Mars Sensor Networks, IEEEAC. 1165-1178(2002).

4. Ian D. Chakeres, Eliszbeth M. Belding-Royer. AODV Routing Protocol Implementation Design,.In Proceedings of 24th International Conference on Distributed Conference on Distributed Computing Systems Workshops(ICDCSW'4). IEEE Computer Society, USA. 698703(2009) .

5. Devu Manikantan Shila, Tricha Anjali. Load aware traffic engineer for mesh networks. Computer Communication.31,1460-1469 (2008).

6. Yunhuai Liu, Lionel Ni. A Generalized Prob-abilistic Topology Control for Wireless Sensor Networks, INFOCOM'09. 698-702(2009).

7. Paolo Santi. Topology Control in Wireless ad hoc and Sensor Networks. Journal ACM Computing Surveys(CSUR). 37,892-899 (2005).

8. Ricardo Silva. Evaluating 6low Pan implementations in WSNs. In Proceedings of the 9th Conference on Computer Networks, Oeiras, Portugal. 15-16(2015).

9. Bart Scheers, Wim Mees, Ben Lauwens. De-velopment on an IEEE802.15.4-based wireless sensor network. Journal of Telecommunication and Information Technology.115-119 (2012).

10. Panneer Muthukumaran, Rodolfo de Paz, Rostislav Spinar, Dirk Pesch. Mesh MAC: Enabling Mesh Networking over IEEE802.15.4 through distributed beacon scheduling. Ad hoc Networks, Lecture Notes of the Institute for Computer Sciences, Social Informatics and Telecommunications Engineering. 28, 561-575(2013).

11. Geoff Mulligan. 6Lo WPAN Working Group, The 6Lo WPAN Architecture, Em Nets 2007. 458-476(2007). 
12. Jamal N. Al-Karaki, Ahmed E Kamal, Routing Techniques in Wireless Sensor Networks: A Survey. Wireless Communications, IEEE. 77-83(2014)..

13. Bahareh Gholamzadeh, Hooman Nabovati. Concepts for Designing Low Power Wireless Sensor Networks. World Academy of Science, Engineering and Technology . 45-49(2014).

14. Philip Levis, Neil Patel, David Culler, Scott Shenker. Trickle: A Self-Regulating Algorithm for Code Propagation and Maintenance in Wireless Sensor Networks. 99-112(2004). 